\title{
Risk of Airway Fire with the use of KTP Laser and High Flow Humidified Oxygen Delivery in a Laryngeal Surgery Model
}

Lucy Huang ( $\square$ lucy.huang.tw@gmail.com )

Flinders University

\section{Adam Badenoch}

Flinders Medical Centre

Marthinus Vermeulen

Flinders Medical Centre

Shahid Ullah

Flinders University

\section{Charmaine Woods}

Flinders University

Theodore Athanasiadis

Flinders Medical Centre

\section{Eng Hooi Ooi}

Flinders University

\section{Research Article}

Keywords: THRIVE, KTP laser, airway fire, high flow oxygen

Posted Date: September 28th, 2021

DOl: https://doi.org/10.21203/rs.3.rs-926775/v1

License: (9) This work is licensed under a Creative Commons Attribution 4.0 International License. Read Full License

Version of Record: A version of this preprint was published at Scientific Reports on January 11th, 2022. See the published version at https://doi.org/10.1038/s41598-021-04636-3. 


\section{Abstract}

\section{Background}

Airway surgery presents a unique environment for operating room fire to occur. This study aims to explore the factors of combustion when using KTP laser with high flow oxygen in an ex-vivo model.

\section{Methods}

The variables tested were varying tissue type, tissue condition, oxygen concentration, laser setting, and smoke evacuation in a stainless-steel model. Outcome measures were time of lasing to the first spark and/or flame. A multivariate Cox proportional hazard model was used to determine the risk of spark and flame across the different risk factors.

Results

For every $10 \%$ increase in oxygen concentration above $60 \%$ the risk of flame increased by a factor of 2.3 . Continuous laser setting at $2.6 \mathrm{~W}$ increased the risk by a factor of 72.8 . The risk of lasing adipose tissue is 7.3 times higher than that of muscle. Charred tissue increases the risk of flame by a factor of 92.8 . Flame occurred without a preceding spark $93.6 \%$ of the time.

Conclusions

Using KTP laser in the pulsed mode with low wattages, minimising lasing time, reducing the oxygen concentration and avoiding lasing adipose or charred tissue produce a relatively low estimated risk of spark or flame.

\section{Introduction}

Operating-room airway fires are serious and potentially fatal complications but fortunately rare in otolaryngologic surgery ${ }^{1}$. Airway fires have been described during adenotonsillectomy, tracheostomy and endoscopic airway surgery ${ }^{2}$. The triad of essential elements required for an airway fire are oxygen, fuel, and an ignition source ${ }^{3}$. Oxygen is in abundance in a shared airway setting where it may be delivered via a facemask, traditional nasal cannulae, supraglottic jet ventilation, endotracheal tube, or high-flow nasal oxygen. The ignition source is usually from the heat generated from electrocautery devices or lasers. Potential fuel sources include endotracheal tubes (ETT), gauze, drapes and the eschar from charred tissue $^{4}$.

Transnasal humidified rapid insufflation ventilatory exchange (THRIVE) is a method of oxygenating patients in a shared airway setting by delivering oxygen at a high flow rate to the lungs without an endotracheal tube. It allows for an unobstructed surgical field and eliminates the endotracheal tube as a potential fuel for ignition. Nasal high-flow oxygen used for THRIVE usually delivers $100 \%$ oxygen, however devices incorporating oxygen-air blenders are now available to alter the oxygen concentration 
delivered. Reports of fires during the use of nasal high flow oxygen in conjunction with electrocautery and laser are also emerging ${ }^{5-7}$.

The use of lasers in laryngeal surgery has increased over recent years, delivering targeted resection with improved haemostasis ${ }^{8}$. The traditionally used carbon dioxide $\left(\mathrm{CO}_{2}\right)$ laser has a wavelength of $10,600 \mathrm{~nm}$, which is strongly absorbed by water and tissue and is associated with most airway fire reports to date $3,9,10$. By contrast, Potassium-titanyl-phosphate (KTP) laser is a solid-state laser with a wavelength of $532 \mathrm{~nm}$, resulting in specific absorption of energy by oxyhaemoglobin in red blood cells, producing photoangiolysis of blood vessels at lower tissue temperature with less vaporisation ${ }^{11,12}$. These factors may theoretically reduce the risk of an airway fire when used in upper airway surgery, compared with the $\mathrm{CO}_{2}$ laser. Studies examining the factors that contribute to the risk of airway fires in clinical practice have largely been conducted using the $\mathrm{CO}_{2}$ laser ${ }^{3,13-15}$. The study by Roy et al. used a mannequin intubated with a laser safe ETT, demonstrating an immediate sustained fire when the $\mathrm{CO}_{2}$ laser struck and perforated the ETT cuff. This occurred with oxygen concentration as low as $40 \%$ indicating that traditional methods of airway management with an ETT for upper airway laser use are not risk free ${ }^{2}$. Dhar et al studied $\mathrm{CO}_{2}$ laser in combination with subglottic jet ventilation in a porcine model, identifying oxygen concentration, laser power and dry fuel source as independent risk factors for combustion ${ }^{13}$. A subsequent study by Stuermer et al. confirmed laser power and oxygen concentration as risk factors and identified tissue type (adipose, cartilage, muscle), tissue quality (fresh, charred) and the use of smoke evacuation as additional independent risk factors for combustion in an ex-vivo plexiglass model using $\mathrm{CO}_{2}$ laser $^{3}$. These two studies were conducted using non-humidified oxygen sources at flow rates significantly lower (jet ventilation at 2 bar and low flow rate at 10L/minute) than those generated when using THRIVE (70L/min) ${ }^{3,13}$. It is possible that $100 \%$ humidification of the gas mixture and the high flow rate provided by THRIVE reduces the risk of combustion ${ }^{15}$.

There has been no study investigating the risk of KTP laser causing an airway fire despite its common use in laryngeal surgery. The aim of this study is to examine the factors associated with combustion when using KTP laser in combination with humidified high-flow nasal oxygen. We developed an ex-vivo model which is designed to simulate laryngeal surgery to facilitate risk prediction of spark and flame under clinically relevant conditions.

\section{Methods}

Human ethics was not required for this study as all experiments were performed using animal tissue (porcine muscle and adipose tissue) purchased from the local butcher. This study is listed on the Animal Register at Flinders University.

The ex-vivo experiment took place in the operating theatre using standard anaesthetic and operating equipment. A model was designed by the Biomedical Engineering Department to simulate the upper airway, consisting of a cylindrical stainless-steel chamber (outer diameter: $10 \mathrm{~cm}$, inner diameter: $5 \mathrm{~cm}$, 
length: $32 \mathrm{~cm}$; Figure $1 \mathrm{a}$ and b). Positioned superiorly, are openings for the Optiflow ${ }^{\mathrm{TM}}$ nasal canula, KTP laser fibre holder, suction, endoscopic camera, and an oxygen sensor to verify precise tissue oxygen concentration at the point of lasing. A plastic covering was used to reduce oxygen loss and maximise oxygen concentration within the chamber. Inferiorly, positioned $25 \mathrm{~cm}$ from the opening is a metal platform that holds the tissue being lasered (Figure 1c). The Aura XP Laser Therapy System ${ }^{\mathrm{TM}}$ (Boston Scientific) was used with EndoStat $0.4 \mathrm{~mm}$ laser fibres, using a non-contact firing technique. Optiflow ${ }^{\mathrm{TM}}$ (Fisher \& Paykel), incorporating an oxygen-air blender, was used to provide flow rates of $70 \mathrm{~L} / \mathrm{minute}$ (min), simulating THRIVE with the ability to alter the oxygen concentration.

Porcine adipose and muscle tissue were used, as they represented the most and least flammable tissue type in the study performed by Stuermer et al ${ }^{3}$. The KTP laser settings were chosen based on clinical relevance ( $2.6 \mathrm{~W}$ continuous, $5 \mathrm{~W}$ continuous, $26 \mathrm{~W}$ pulsed and $35 \mathrm{~W}$ pulsed settings; pulsed setting set at 15 millisecond pulse width, 2 pulse per second). Lasing was performed on both charred and uncharred, adipose and muscle tissue with variable oxygen concentrations $(30 \%, 40 \%, 50 \%, 60 \%, 70 \%, 80 \%, 90 \%)$, with and without smoke evacuation. Each piece of tissue was placed in a plastic bag and warmed using a water bath to $30^{\circ} \mathrm{C}$. Charring of tissue was conducted using a kitchen blow torch to enable even charring on tissue surface (Figure 2).

$100 \%$ humidification and gas flow rate at $70 \mathrm{~L} / \mathrm{min}$ were used in every instance. Each combination of variables was repeated at least five times to ensure reproducibility. If a flame occurred, oxygen was immediately reduced to $21 \%$ using the oxygen-air blender while maintaining $70 \mathrm{~L} / \mathrm{min}$ flow rates, which rapidly extinguished the flame. Example videos have been provided in Supplementary video 1 and 2.

\section{Outcome measures}

The outcomes were the occurrence of a spark and/or flame. Flame is considered the most important patient-centred outcome; however, sparks are often used clinically as a surrogate marker to indicate increased risk of flame if lasing continues, therefore spark and flame were modelled separately from the same data set. Time was measured from the start of lasing to the time to first spark/flame, or until 60 seconds. A 60 second cut off time was used, as lasing beyond 60 seconds is not clinically relevant.

\section{Statistical methods}

A multivariate Cox proportional hazard model was used to examine the risk of spark/flame across tissueoxygen concentration, laser setting, tissue type, charring and smoke evacuation. Time to event was measured from the time of lasing onset to the time of spark/flame, experiments were censored at 60 seconds. As each combination of experimental conditions was repeated 5 times the models captured the mixed effect using shared frailty - the same random effect is shared by all repeated events within the same experimental group. The frailties are assumed to be gamma-distributed latent random effects that affect the hazard multiplicatively. The variables included in the final multivariate model were oxygen concentration, laser setting, tissue type and charring. The inclusion criterion was based on clinical importance, and statistical significance at $\mathrm{P} £ 0.20$ from a univariate model. Variables tested 
experimentally and included in the univariate analyses were all previously identified as independent risk factors in previous studies using different laser types ${ }^{3,15}$. Smoke evacuation was subsequently excluded from the multivariable model based on non-significance in the univariate model. Additionally, re-inclusion of smoke evacuation into the final model did not result in a statistically significant association or significant change in the model coefficients. Proportional hazard assumption was tested by log-log plot

of survival and Schoenfeld residuals and was found to be valid. Linear splines were employed to account for non-linearity of association between oxygen concentration and outcome hazard. The spline cut-off at $60 \%$ oxygen was selected based on it being an inflection point in the observed data and a judgement that this cut-off was clinically relevant in terms of the risk of hypoxia when THRIVE is used clinically. Cumulative hazard curves were evaluated by standard Nelson-Aalen cumulative hazard functions.

The clinically relevant probabilities of spark or flame were established using the final multivariable Cox proportion hazard models by restricting the predictive model to 5 seconds of lasing (or time to event if $<5$ seconds) to facilitate a pragmatic and clinically relevant risk assessment. Probabilities were generated based on calculation of coefficients from the regression model and estimates of 5-second risk of spark or flame. In particular, the coefficient was multiplied by the value of the variable and then summed for each variable to get the scores. The baseline survival function was then exponentiated by the score and then subtracted from 1 to calculate the 5-second risk. Finally, the adjusted predictions were displayed after using a restricted cubic spline to account for non-linear relationships between oxygen concentration and the risk of spark or flame.

Model diagnostics and goodness of fit were evaluated by Harrell's $\mathrm{C}$ concordance statistic. The two-sided test was performed for all analysis and the level of significance was set at $p<0.05$.

\section{Results}

The ex-vivo testing resulted in 2,182 firing episodes across a total of 224 combinations of variables. There were no missing data.

Flames were preceded by sparks in $6.4 \%$ of instances and occurred without sparks $93.6 \%$ of the time. In those instances where sparks preceded flames the median (25th -75th percentiles) time difference between spark and flame was 19 (4-44) seconds.

Notably, no sparks or flames were observed when laser firing was limited to 5 seconds at fresh tissue irrespective of oxygen concentration, laser setting or tissue type.

Oxygen concentration, laser mode, tissue type, and tissue quality were all independent risk factors for spark and flame (Fig. 3a-d). Smoke evacuation was not a significant predictor of spark nor flame in either the univariate or multivariate analyses and was therefore excluded from the final multivariable model (Fig. 3e). Results of the final multivariable model for spark and flame are presented in (Fig. 4). The Harrell's C concordance statistic for the multivariable model was 0.91 for spark and 0.94 for flame. 
When compared with uncharred tissue, the risk $(95 \% \mathrm{Cl})$ of spark when lasing at charred tissue was increased by $34.9(16.8-72.5)[p<0.001]$ and the risk of flame increased by $92.8(31.8-270.4)[p<0.001]$ (Fig. 4(b)).

For oxygen concentration within the range $25-59 \%$, the risk $(95 \% \mathrm{Cl}$ ) of spark increased by $3.9(2.4-6.3)$ $[p<0.001]$ and flame increased by $6.6(3.0-14.5)[p<0.001]$ for every $10 \%$ increase in oxygen concentration. For oxygen concentration within the range $60-90 \%$, the risk of spark increased by 1.8 $(1.3-2.4)[p<0.001]$ and flame increased by $2.3(1.6-3.4)[p<0.001]$ for every $10 \%$ increase in oxygen concentration.

Compared with pulsed 26W (lowest power setting), pulsed 35W increased the risk $(95 \% \mathrm{Cl})$ of spark by $5.7(2.0-15.7)[\mathrm{p}<0.01]$ and the risk of flame by $4.8(1.2-18.6)(\mathrm{p}<0.05)$. The risk $(95 \% \mathrm{Cl})$ of spark at continuous 2.6W and 5W are increased by $28.8(10.0-83.2)[p<0.001)]$ and $48.6(17.0-138.8)[p<0.001]$ respectively. The risk $(95 \% \mathrm{Cl})$ of flame at continuous $2.6 \mathrm{~W}$ and $5 \mathrm{~W}$ are increased by $72.8(17.6-300.5)(\mathrm{p}$ $<0.001)$ and $47.1(12.0-185.7)[p<0.001]$ respectively. There was no statistically significant difference between the risk of spark or flame associated with $2.6 \mathrm{~W}$ and $5 \mathrm{~W}$ continuous settings when compared with each other.

Compared with muscle, the risk $(95 \% \mathrm{Cl})$ of spark when lasing at adipose tissue is $7.3(3.6-14.8)[\mathrm{p}<$ $0.001]$ times higher and the risk of flame is $13.5(5.0-36.1)[p<0.001]$ times higher.

\section{Clinical Risk Estimates}

When modelling specific, clinically relevant scenarios, the risk $(95 \% \mathrm{Cl})$ of flame estimated when using $26 \mathrm{~W}$ pulsed settings with $40 \%$ oxygen was $0.0003 \%$ ( 0 to 0.002 ) (Fig. $5 \mathrm{a}$ ) on uncharred muscle and $0.001 \%$ ( 0 to 0.05 ) on uncharred adipose tissue (Fig. $5 \mathrm{~b}$ ). As a comparison, the most flammable combination ( $5 \mathrm{~W}$ continuous setting on charred adipose tissue) demonstrated the likelihood of flame within 5 seconds is as high as $0.2 \%$ (0-1.6) with $40 \%$ oxygen and $5.7 \%$ (0-56.7) with $60 \%$ oxygen (Fig. 5 c). The inflection point for increased risk of flame with increasing oxygen varies depending on the specific combination of variables (laser mode, tissue type \& charred status) (Fig. 5).

Similar estimates were determined for the occurrence of spark (Supplementary figure S1). The least flammable combination (laser at 26W pulsed setting, on uncharred muscle) (Supplementary figure S1a) resulted in an estimated risk $(95 \% \mathrm{Cl})$ ranging from $0.01 \%(0-0.04)$ at $40 \%$ oxygen to $0.09 \%(0-0.5)$ at $60 \%$ oxygen. The most flammable combination (laser at $5 \mathrm{~W}$ continuous setting, on charred adipose tissue) result in an estimated risk $(95 \% \mathrm{Cl})$ ranging from $1.8 \%(0-9.8)$ at $40 \%$ oxygen to $20.6 \%(0-100)$ at $60 \%$ oxygen.

\section{Discussion}

This is the first study to evaluate the risk of fire when using KTP laser in a high flow oxygen setting. Theoretically fire risks are reduced when THRIVE is used with the removal of potential fuel sources, the 
humidification of gases within the surgical field and the provision of an effective mechanism to remove laser smog. Furthermore, KTP laser has theoretical advantages over $\mathrm{CO}_{2}$ laser in terms of reduced tissue heating dynamics ${ }^{8}$. However, this study demonstrates that these factors do not negate the possibility of an airway fire. Factors comprising of continuous laser mode, oxygen concentration greater than $60 \%$, increased adiposity and the presence of char provide increased risk estimates of spark or flame, with many instances of flame occurring without a preceding spark. Native tissue that is high in adiposity or has been charred during the surgical process are highly combustible, and therefore a high level of vigilance is required of the surgeon to remove any charring when lasing with THRIVE.

Oxygen concentration is an important predictor of the risk of spark and flame. An important clinical question is whether the oxygen concentration required to prevent airway fires is compatible with adequate patient oxygenation. The current recommendation from the Joint Commission on Accreditation of Healthcare Organizations is to use an oxygen concentration of less than $30 \%$ when delivered in an open manner during facial surgery ${ }^{16}$. Despite this recommendation, Roy \& Smith were able to ignite a nonsustained flame when their laser struck and perforated the cuff of a laser safe endotracheal tube, using $5 \mathrm{~W} \mathrm{CO}_{2}$ laser with an oxygen concentration of $29 \%$ for an unspecified length of time ${ }^{2}$. Importantly, the wider pulse width of the KTP laser allows slower heating, providing a theoretical smaller risk of combustion compared to $\mathrm{CO}_{2}$ laser $^{8}$. The current study demonstrates that clinically relevant combinations of oxygen concentration, laser setting and lasing duration result in reasonably low estimated risks; but these may still be considered too high in the clinical setting given the devastating consequences if airway fire is to occur. For example, an estimated risk of flame is $0.05 \%$ ( 5 fires per 10,000 lasing) with an upper limit of the $95 \% \mathrm{Cl}$ of $0.5 \%$ ( 5 fires per 1,000 lasing) at $50 \%$ oxygen with a $26 \mathrm{~W}$ pulsed setting on uncharred adipose tissue. This risk is reduced when oxygen concentration is reduced to $40 \%$ (risk of $0.001 \%$ with an upper limit of $95 \% \mathrm{Cl}$ of $0.005 \%$ ) or if the tissue type is uncharred muscle (estimated risk of $0.003 \%$ with upper limit of $95 \% \mathrm{Cl}$ of $0.03 \%$ ). The oxygen concentration delivered during KTP laser use should be titrated according to patient's oxygen requirements with the potential for delivering higher oxygen concentration when using pulsed KTP settings of lower power. We did not observe any sparks or flames when lasing for less than 5 seconds on uncharred tissue irrespective of oxygen concentration, laser power or tissue type. This indicates that KTP laser and the absence of a fuel source, such as an ETT, reduces the risk of combustion. Furthermore, lasing for less than 5 seconds at a time is highly unlikely to result in combustion. The consequences of an airway fire are severe; therefore, surgeons and anaesthetists should work together with the aim to minimise this risk as much as possible while balancing the risk of hypoxia. An oxygen-air blender can rapidly alter oxygen concentration, providing low oxygen fractions during periods of lasing. Further in-vivo studies are needed to determine the oxygen concentration at the level of the larynx when using an oxygen-air blender with THRIVE.

Smoke evacuation using suction was identified as an important factor to reduce combustion of vaporised carbonised tissue (i.e. laser smog) when using $\mathrm{CO}_{2}$ laser ${ }^{3}$. However, smoke evacuation was not demonstrated as an important factor in the current study. This may reflect the different tissue absorption 
characteristics of KTP laser with reduced surface temperature, resulting in less tissue vaporisation compared to the $\mathrm{CO}_{2}$ laser ${ }^{8}$. Alternatively, the high flow rate of THRIVE at 70L/minute may also mean additional smoke evacuation via suction is redundant. In addition to removing laser smog, a high flow rate may also provide continuous positive pressure to reduce atelectasis and shunting of deoxygenated blood through the lungs when used in-vivo which may maintain oxygenation when using lower oxygen concentration. This is demonstrated in one previous study that an oxygen flow rate of $50 \mathrm{~L} / \mathrm{min}$ delivered via the nose is able to provide a positive airway pressure of $7 \mathrm{cmH}_{2} \mathrm{O}^{17}$.

Spark is commonly used as a warning sign of flame; however, it is important to note that most flames occurred spontaneously without a preceding spark. When a spark did precede a flame, the time between spark and flame was highly variable, often less than 4 seconds. This suggests that sparks should not be used as a reliable indicator of imminent risk of sustained flame.

The continuous laser settings of $2.6 \mathrm{~W}$ and $5 \mathrm{~W}$ produced dangerously high clinical risk estimates of spark and flame. Despite $2.6 \mathrm{~W}$ appearing to be of higher risk than $5 \mathrm{~W}$ there was no statistical difference in the magnitude of these risk estimates with significant overlap of the $95 \%$ confidence interval. Regardless of the estimated clinical risk presented in this study, it would not be advisable to use either of these settings when using KTP laser in conjunction with THRIVE.

The flames that occurred in this study were all successfully extinguished by turning the oxygen concentration down to $21 \%$ using the oxygen-air blender except for one episode where embers caught a piece of dry gauze underneath the apparatus. This was rapidly extinguished with water. This serves as a reminder that reducing the concentration of oxygen along with adequate safety preparation prior to commencing laser airway surgery are important safety steps in the event of an airway fire. It was also noted that when flame was ignited on charred tissue, it rapidly spread to other sections of the tissue. The spread of the flame can be prevented by removing charred tissue from within the surgical field.

\section{Limitations}

This model does not fully simulate a human respiratory system with gases entering the lungs and exiting along the same pathway (trachea), as most of the gas in this system will escape from the inferior end of the model. It also does not simulate the gas absorption that occurs in the human lungs. Therefore, ascertaining the concentration of oxygen at the level of the patient's larynx during upper airway procedures using THRIVE is an important next step to translate this data into meaningful clinical recommendations.

It would not be ethical nor practical to perform this study using live animals with the risk of airway fires and number of repeat experiments (> 2000 lasing) done to ensure reproducibility. Porcine tissue is frequently used as an analogue for human tissue in forensic sciences ${ }^{18}$ and were used instead of human larynges. This study chose the most and least flammable tissue types, allowing us to present the range of risks associated with using KTP laser in this setting. The flammability characteristics of the surface 
mucous membrane during laryngeal surgery will be different to either adipose or muscle tissue, but will fall within the described range of risk prediction. Each porcine specimen may not have the exact same consistency of adiposity or muscle for each firing, but the chosen specimens were largely of the same tissue type macroscopically. KTP laser has an affinity for oxyhaemoglobin and as the tested tissue is non-living and has no circulating blood, this aspect could not be tested.

\section{Conclusion}

There remains a high risk of combustion when using KTP laser in a humidified oxygen rich environment especially with high oxygen concentration, charring and adiposity. Extreme caution should be taken to reduce or eliminate these factors when using KTP laser in a shared airway setting. When combining high flow oxygen with KTP laser for upper airway surgery with standard clinical equipment in a real operating room environment the following combinations provided low estimated risk of spark or flame: KTP laser in the pulsed mode with low wattages, minimising lasing time, reducing the oxygen concentration and avoiding lasing adipose or charred tissue. Sparks should not be relied on as reliable indicators of imminent flame.

\section{Declarations}

\section{Acknowledgements}

We acknowledge and thank Olivia Lockwood and Roy Broughton at Flinders Medical Centre Biomedical Engineering Department for their expertise developing the stainless-steel model.

\section{Authors' contribution}

LH designed the analysis, collected the data, interpreted the data and drafted the manuscript. $A B$ designed the analysis, collected the data, analysed and interpreted the data and drafted the manuscript. MV collected the data and edited the manuscript. SU performed the statistical analysis, assisted with interpretation of the analyses and drafted the manuscript. CW conceived and designed the analysis, interpreted the data and drafted the manuscript. TA conceived and designed the analysis, collected the data, sourced equipment, interpreted the data and drafted the manuscript. EHO conceived and designed the analysis, collected the data, sourced equipment, interpreted the data and drafted the manuscript. All authors contributed to the writing of the manuscript and approved the final manuscript.

\section{Competing interests}

The authors declare that they have no competing interests. 


\section{Data availability}

The datasets used and analysed during the current study are made available via the link provided.

\section{Ethics declarations}

Not applicable

\section{Consent to publish}

Not applicable

\section{References}

1. Day, A. T., Rivera, E., Farlow, J. L., Gourin, C. G. \& Nussenbaum, B. Surgical Fires in Otolaryngology: A Systematic and Narrative Review. Otolaryngol Head Neck Surg, 158, 598-616 https://doi.org/10.1177/0194599817746926 (2018).

2. Roy, S. \& Smith, L. P. Surgical fires in laser laryngeal surgery: are we safe enough? Otolaryngol Head Neck Surg, 152, 67-72 https://doi.org/10.1177/0194599814555853 (2015).

3. Stuermer, K. J., Ayachi, S., Gostian, A. O., Beutner, D. \& Huttenbrink, K. B. Hazard of CO(2) laserinduced airway fire in laryngeal surgery: experimental data of contributing factors. Eur Arch Otorhinolaryngol, 270, 2701-2707 https://doi.org/10.1007/s00405-013-2521-1 (2013).

4. Yardley, I. E. \& Donaldson, L. J. Surgical fires, a clear and present danger. The Surgeon, 8, 87-92 https://doi.org/10.1016/j.surge.2010.01.005 (2010).

5. Greenland, K. B., Stokan, M. J. \& Culwick, M. D. Operating theatre fires: Adding more oxygen to the mix. Anaesth Intensive Care, 47, 399-400 https://doi.org/10.1177/0310057x19860976 (2019).

6. Onwochei, D., El-Boghdadly, K., Oakley, R. \& Ahmad, I. Intra-oral ignition of monopolar diathermy during transnasal humidified rapid-insufflation ventilatory exchange (THRIVE. Anaesthesia, 72, 781783 https://doi.org/10.1111/anae.13873 (2017).

7. Chang, M. Y. et al. Fire safety study on high-flow nasal oxygen in shared-airway surgeries with diathermy and laser: simulation based on a physical model. J. Clin. Monit. Comput, https://doi.org/10.1007/s10877-021-00690-4 (2021).

8. Yan, Y. et al. Use of lasers in laryngeal surgery. J Voice, 24, 102-109 https://doi.org/10.1016/j.jvoice.2008.09.006 (2010).

9. Santos, P., Ayuso, A., Luis, M., Martinez, G. \& Sala, X. Airway ignition during $\mathrm{CO} 2$ laser laryngeal surgery and high frequency jet ventilation. Eur J Anaesthesiol, 17, 204-207 (2000).

10. Sosis, M. B. Airway fire during co2 laser surgery using a Xomed Laser endotracheal tube., 72, 747749 https://doi.org/10.1097/00000542-199004000-00027 (1990). 
11. Yan, Y. et al. Use of Lasers in Laryngeal Surgery. Journal of Voice, 24, 102-109 https://doi.org/10.1016/j.jvoice.2008.09.006 (2010).

12. Broadhurst, M. S. et al. Effects of $532 \mathrm{~nm}$ pulsed-KTP laser parameters on vessel ablation in the avian chorioallantoic membrane: implications for vocal fold mucosa., 117, 220-225 https://doi.org/10.1097/mlg.0b013e31802b5c1c (2007).

13. Dhar, V. et al. Impact of oxygen concentration and laser power on occurrence of intraluminal fires during shared-airway surgery: an investigation. J. Laryngol. Otol, 122, 1335-1338 https://doi.org/10.1017/S0022215108003101 (2008).

14. Roy, S. \& Smith, L. P. What does it take to start an oropharyngeal fire? Oxygen requirements to start fires in the operating room. Int J Pediatr Otorhinolaryngol, 75, 227-230 (2011).

15. Takanashi, S. et al. Airflow through the auxiliary line of the laser fiber prevents ignition of intra-airway fire during endoscopic laser surgery. Lasers Surg Med, 31, 211-215 https://doi.org/10.1002/lsm.10097 (2002).

16. Lampotang, S., Gravenstein, N., Paulus, D. A. \& Gravenstein, D. Reducing the incidence of surgical fires: supplying nasal cannulae with sub-100\% 02 gas mixtures from anesthesia machines. Anesth Analg, 101, 1407-1412 https://doi.org/10.1213/01.Ane.0000180215.50589.02 (2005).

17. Ritchie, J. E., Williams, A. B., Gerard, C. \& Hockey, H. Evaluation of a humidified nasal high-flow oxygen system, using oxygraphy, capnography and measurement of upper airway pressures. Anaesth Intensive Care, 39, 1103-1110 https://doi.org/10.1177/0310057x1103900620 (2011).

18. Matuszewski, S. et al. Pigs vs people: the use of pigs as analogues for humans in forensic entomology and taphonomy research. Int J Legal Med, 134, 793-810 https://doi.org/10.1007/s00414-019-02074-5 (2020).

\section{Figures}




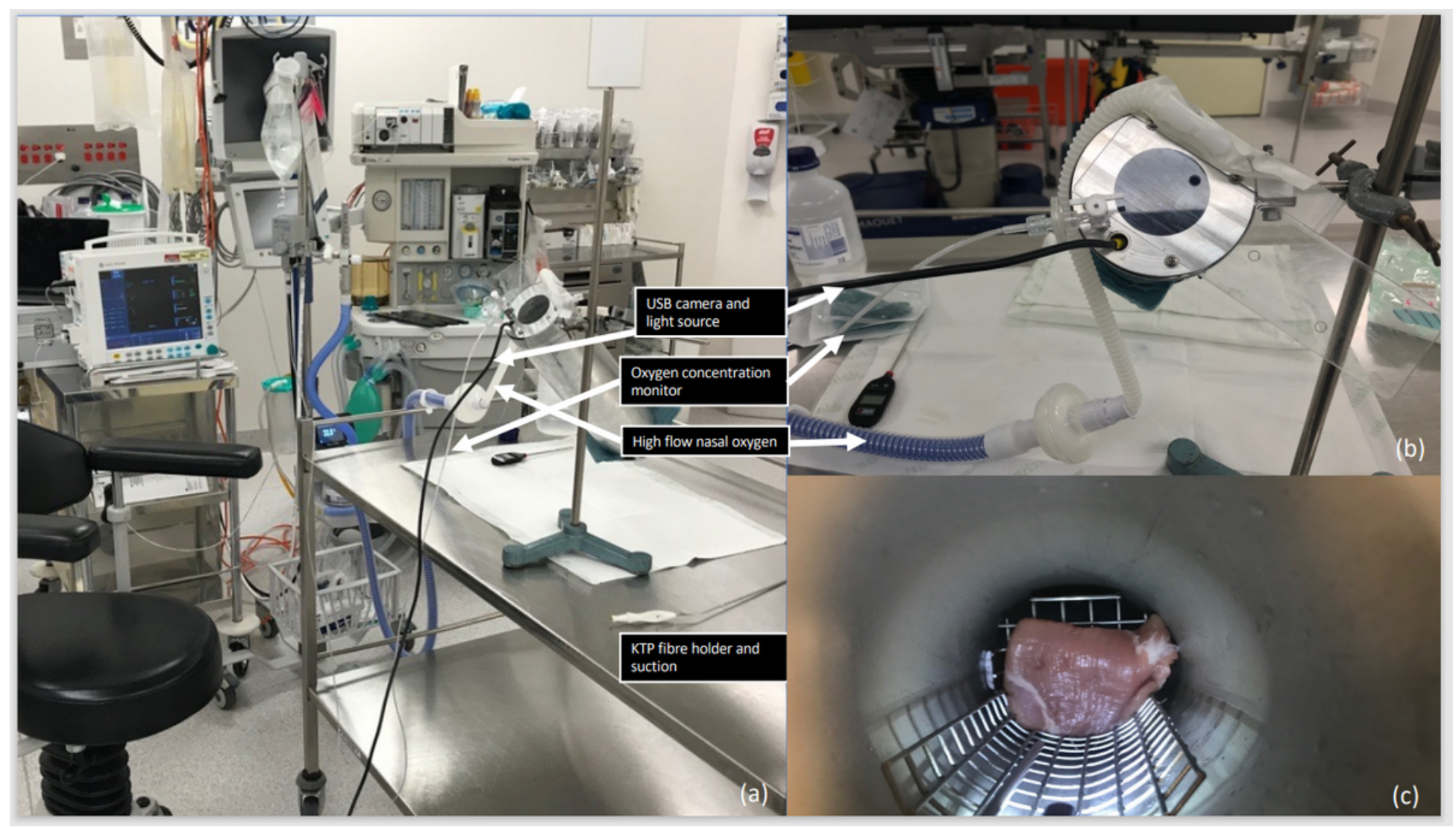

\section{Figure 1}

Experimental setup (a) Experimental setup with the stainless steel laryngeal model in an operating theatre environment with OptiflowTM; (b) The inlet of the laryngeal model with a plastic covering, OptiflowTM nasal cannula, oxygen sensor probe and USB camera; (c) The internal view of the model with a stainlesssteel platform holding the porcine specimen.

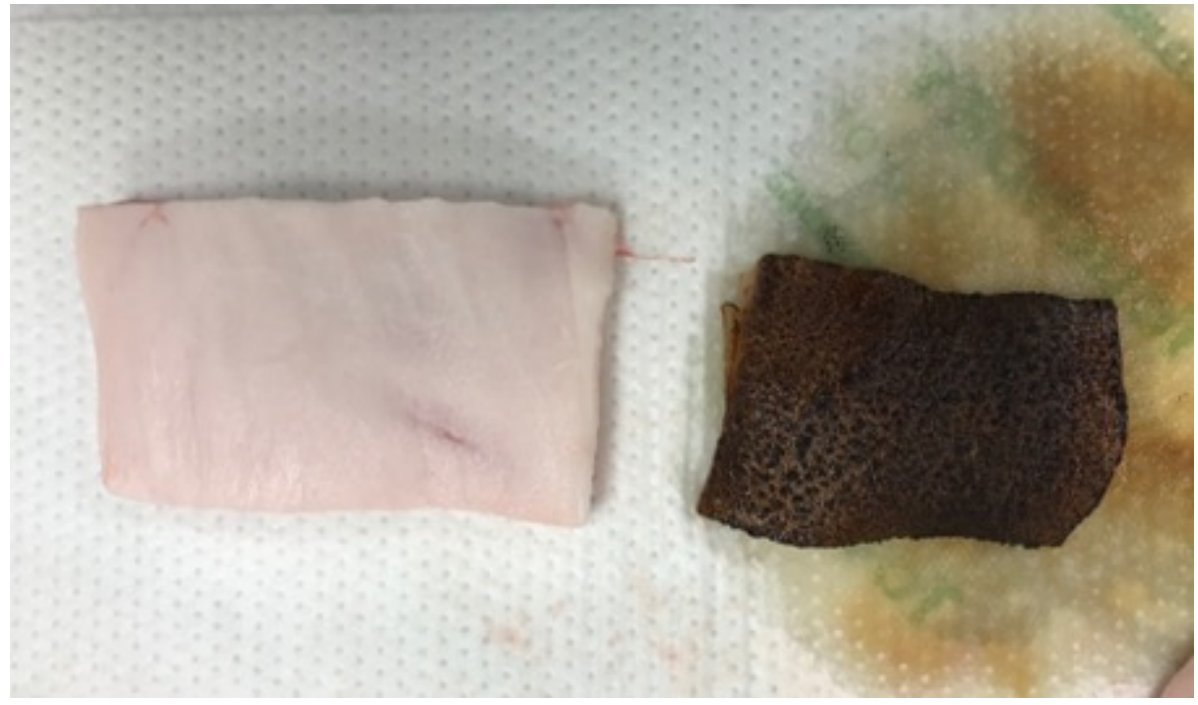

Figure 2

An example of fresh and charred adipose tissue. 
(a)

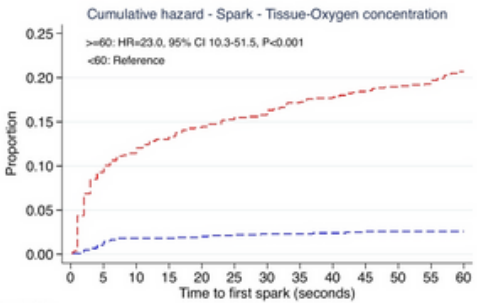

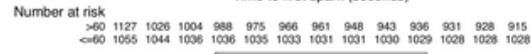
$--\cdots>60 \quad-\cdots<60$

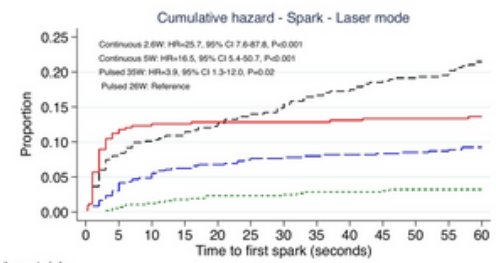

(b)
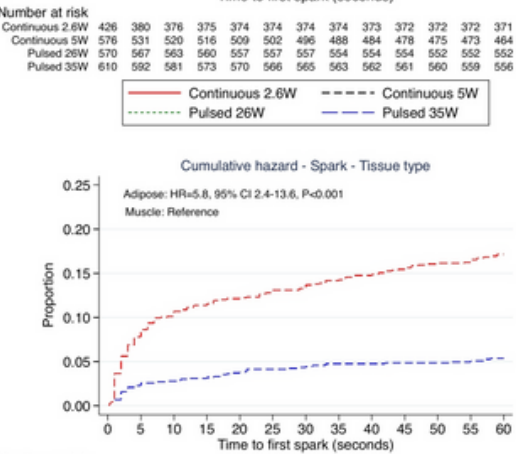

(c)
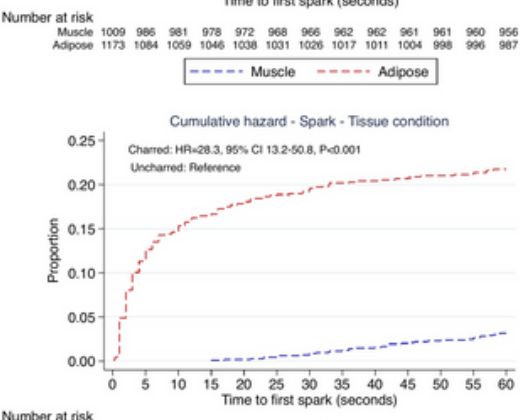

(d)

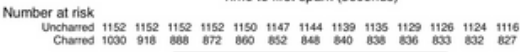

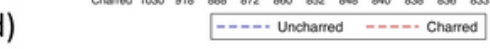

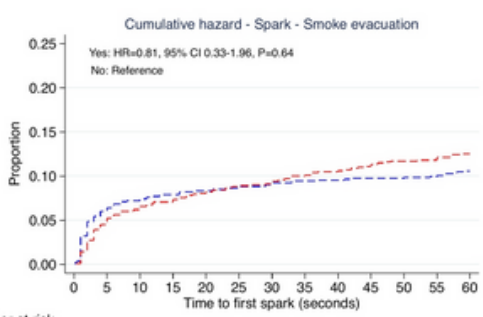

(e)

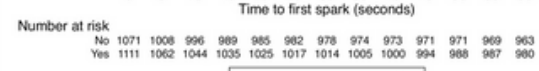

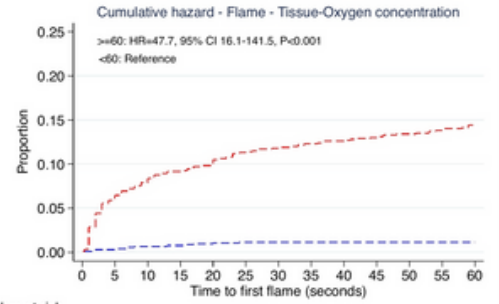

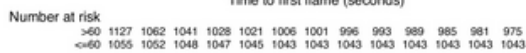

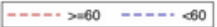

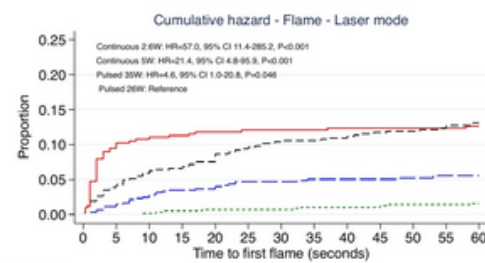

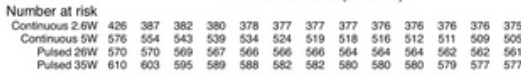
Continuous 2.6W $-\cdots$ Continuous $5 \mathrm{~W}$

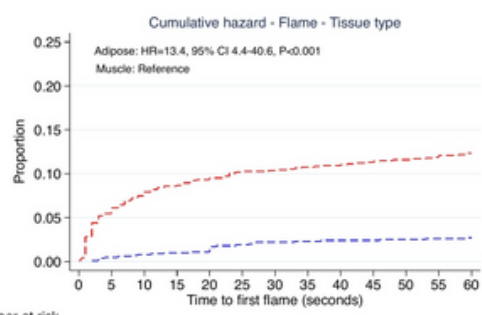

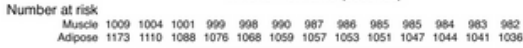
----- Muscle ----- Adipose

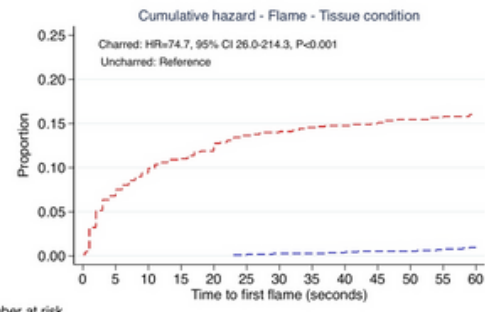

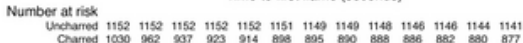
--.-. Uncharred --.-- Charred

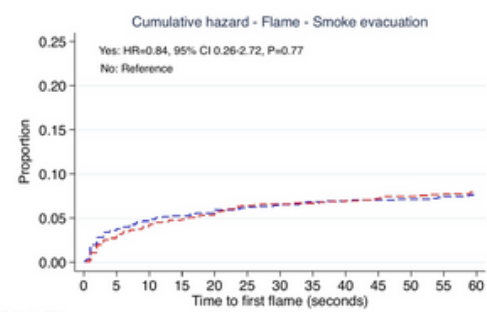

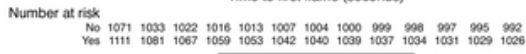

\section{Figure 3}

Nelson-Aalen cumulative hazard function of spark and flame by (a) oxygen concentration (b) laser mode (c) tissue type (d) tissue condition (e) smoke evacuation based on ex-vivo experimental data 
(a)

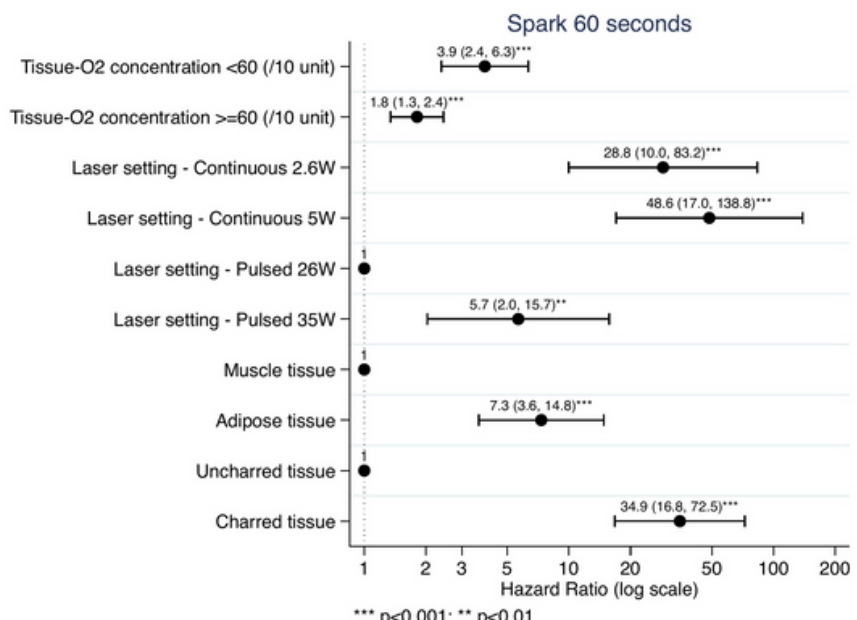

(b)
Flame 60 seconds

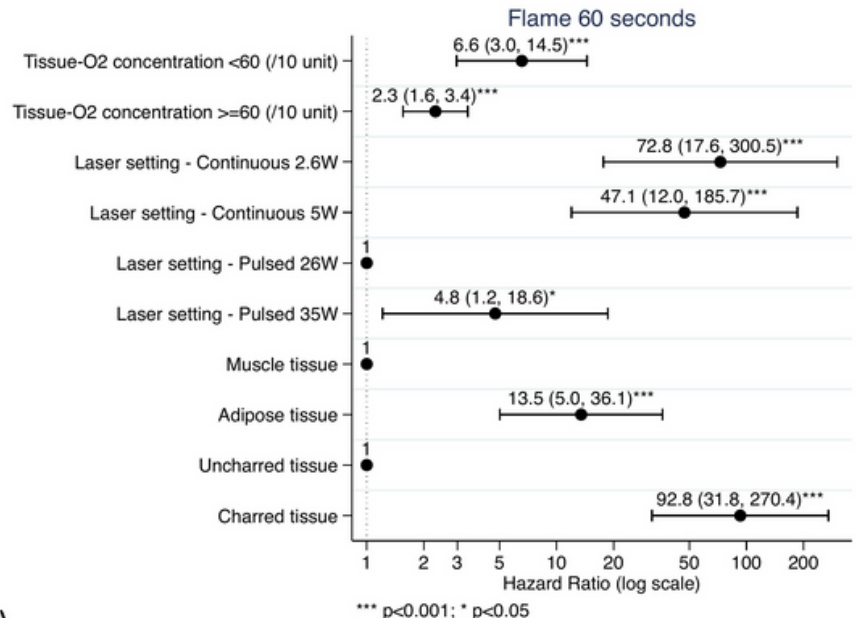

\section{Figure 4}

Coefficient plot from multivariate Cox proportional hazard models of (a) spark and (b) flame censored after 60 seconds from ex-vivo experimental data. 
(a)
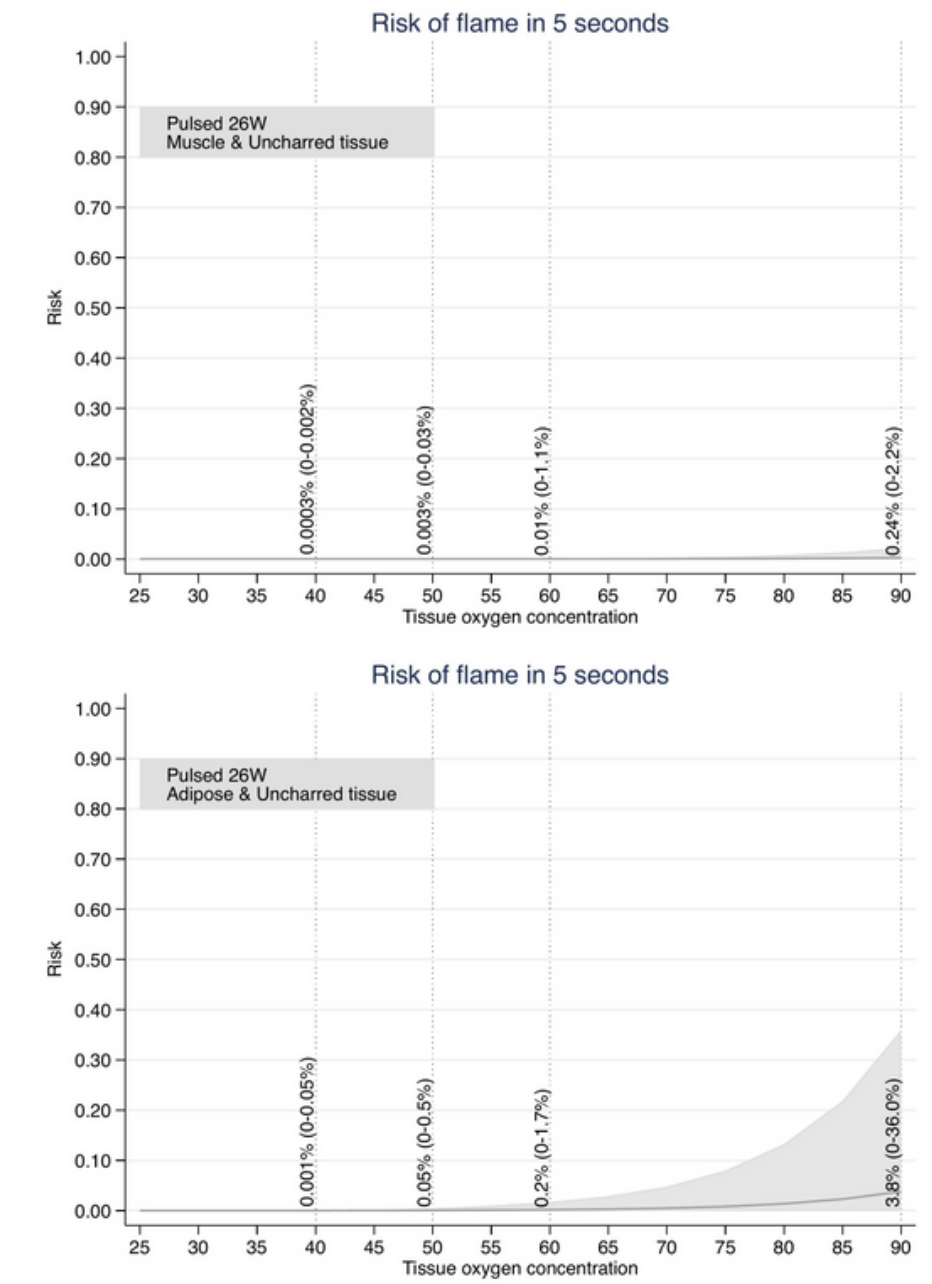

(b)

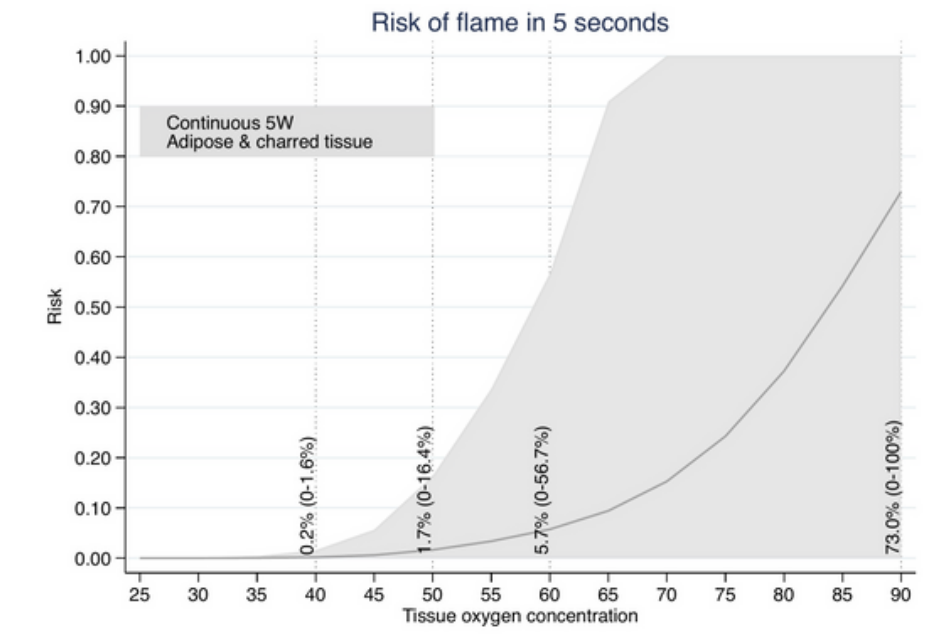

(c)

\section{Figure 5}

Estimated clinical risk of flame when lasing up to 5 seconds by oxygen concentration, laser mode, tissue type and charring based on experimental data. Percentages on the figures show the risks for oxygen concentration 40, 50, 60, and 90 respectively. (a) Laser setting: $26 \mathrm{~W}$ pulsed, tissue: uncharred muscle; (b) Laser setting: 26W pulsed, tissue: uncharred adipose tissue; (c) Laser setting: $5 \mathrm{~W}$ continuous, tissue: charred adipose tissue. 


\section{Supplementary Files}

This is a list of supplementary files associated with this preprint. Click to download.

- SupplementaryFigureS1.jpg

- SupplementaryVideo1.mov

- SupplementaryVideo2.mov

- SupplementaryInfoFile.docx 